\title{
Comparative effect of tacrolimus $0.1 \%$ ointment and clobetasol $0.05 \%$ ointment in patients with oral lichen planus
}

Corrocher G, Di Lorenzo G, Martinelli N, Mansueto P, Biasi D, Nocini PF, Lombardo $G$, Fior A, Corrocher R, Bambara LM, Gelio S, Pacor ML. Comparative effect of tacrolimus $0.1 \%$ ointment and clobetasol $0.05 \%$ ointment in patients with oral lichen planus. J Clin Periodontol 2007; 35: 244-249. doi: 10.1111/j.1600-051X.2006.01191.x.

\section{Abstract}

Background: Oral lichen planus (OLP) is considered to be an autoimmune disease of unknown aetiology that affects the mucosae, especially the oral cavity.

Objective: We compared tacrolimus $0.1 \%$ ointment and clobetasol $0.05 \%$ ointment for the treatment of OLP.

Patients and Methods: A total of 32 patients (20 females and 12 males; all white, Italian origin, mean age of $43.6 \pm 18.4$ years; 16 patients per treatment group) were treated with tacrolimus or clobetasol ointment for 4 weeks in a randomized,

double-blind, clinical trial. Pain severity, burning sensation, and mucosal lesion extension were assessed using a four-point scale.

Results: At the end of the treatment period, symptom scores were significantly lower in the tacrolimus group than in the clobetasol group.

Conclusion: The results of this study suggest that tacrolimus $0.1 \%$ ointment is more effective than clobetasol propionate $0.05 \%$ ointment in the treatment of OLP.

However, other studies are needed to confirm the effectiveness of this treatment before it can be recommended for use in clinical practice.

\author{
Giovanni Corrocher ${ }^{1}$, Gabriele Di \\ Lorenzo $^{2}$, Nicola Martinelli ${ }^{3}$, \\ Pasquale Mansueto ${ }^{2}$, Domenico \\ Biasi $^{3}$, Pier Francesco Nocini ${ }^{1}$, \\ Giorgio Lombardo ${ }^{1}$, Andrea Fior ${ }^{1}$, \\ Roberto Corrocher ${ }^{3}$, Lisa Maria \\ Bambara $^{3}$, Stefano Gelio 4 and \\ Maria Luisa Pacor ${ }^{3}$ \\ ${ }^{1}$ Dipartimento di Scienze \\ Morfologico-Biomediche, Sezione di \\ Chirurgia Maxillo-Faciale \\ e Odontostomatologia, Università di Verona, \\ Verona, Italy; ${ }^{2}$ Dipartimento di Medicina \\ Clinica e delle Patologie Emergenti, \\ Università di Palermo, Palermo, Italy; \\ ${ }^{3}$ Dipartimento di Medicina Clinica \\ e Sperimentale, ${ }^{4}$ Servizio Farmacia del \\ Policlinico "G. B. Rossi”, Università \\ di Verona, Verona, Italy
}

Key words: clobetasol; oral lichen planus; tacrolimus

Accepted for publication 29 November 2007
Lichen planus is a chronic inflammatory disorder of cutaneous and mucosal tissues that is estimated to affect $0.5-2.2 \%$ of the general population (DeRossi $\&$ Ciarrocca 2005). Oral lichen planus

Conflict of interest and source of
funding statement
The authors declare that they have no
conflict of interests.
This study was supported by grants from
the Ministero Italiano University of
Ricerca, Rome, Italy, to Prof. M. L. Pacor
and Prof. G. Di Lorenzo. Funding was
used to purchase study drugs and software.
Clobetasol propionate $0.5 \%$ ointment was
purchased from GlaxoSmithKline,
Verona, Italy.

(OLP) is considered to be an autoimmune disease of unknown aetiology in which epithelial cells are recognized as foreign due to changes in cell surface antigenicity (Edwards \& Kelsch 2002). In contrast to cutaneous lichen planus, in which the clinical course is often mild and resolves within 2 years, mucosal OLP tends to follow a more chronic course and is often accompanied by acute exacerbations characterized by pain and burning sensations with considerable loss of quality of life (Dissemond 2004). An epidemiological study demonstrated that women suffer from OLP more frequently $(75 \%)$ than men $(25 \%)$, and that the disease is more frequent in people over 40 years of age (Eisen 2002). The diagnosis of OLP is based on a combination of characteristic clinical findings and histopathological examination. Three different forms of OLP are currently described: (1) a hyperkeratotic (white) form that is often asymptomatic, (2) an atrophic or erythematous (red) form that is associated with mild to moderate symptoms, and (3) an erosive or ulcerative (yellow) form that usually has persistent symptoms (Setterfield et al. 2000, de Moura Castro Jacques et al. 2003, Eisen 2003, Lodi et al. 2005).

Patients with symptomatic OLP often require intensive therapy to reduce the signs and symptoms of this painful and disabling inflammatory disease (Chan et al. 2000). When pain and burning sensations are severe, therapeutic options 
include high-potency topical corticosteroids; systemic corticosteroids may be indicated in patients whose condition is unresponsive to topical corticosteroids. For such patients, other treatments include immunosuppressive drugs such as cyclosporine, tacrolimus, and retinoids (Sloberg et al. 1983, Rozycki et al. 2002, Carbone et al. 2003, Conrotto et al. 2006).

The aim of this randomized, doubleblind, 4-week clinical trial was to compare treatment efficacy and tolerability of topical tacrolimus $0.1 \%$ ointment with topical clobetasol propionate $0.05 \%$ ointment in adults with OLP.

\section{Patients and Methods Patients}

This study was approved by the Human Subjects Committee of the Institutional Review Board of the Dipartimento di Medicina Clinica e Sperimentale, Università di Verona, Verona, Italy, where the study was conducted. All patients provided written informed consent to participate, in compliance with the institutional policy of Policlinico "GB Rossi", of Verona, Italy. The study was conducted in accordance with the principles of the Declaration of Helsinki (World Medical Association Declaration of Helsinki 1989) and Good Clinical Practice (European Agency for the Evaluation of Medicinal Products 2002).

\section{Inclusion and exclusion criteria}

Patients aged $>18$ years were selected from the electronic medical records of the Dipartimento di Medicina Clinica e Sperimentale, Università di Verona, Verona, Italy on the basis of a clinical history of moderate to severe OLP. The clinical diagnosis was confirmed histologically in all patients by one of the investigators (G. C.) using the following features of OLP: (1) liquefaction of oral epithelium, (2) apoptosis of keratinocytes, (3) focal areas of hyperkeratinized epithelium, and (4) presence of a dense band-like lymphocytic infiltrate at the interface between the epithelium and connective tissue (Edwards \& Kelsch 2002). The exclusion criteria were presence of skin and/or genital lesions, histopathologic signs of dysplasia, treatment with drugs that may induce lichenoid reactions, previous treatment with systemic corticosteroids, immunosuppressant therapy, topical glucocorticoids or tacrolimus within the previous 8 weeks, chronic liver disease, immune system dysfunction, haematological disease, pregnancy, and lactation.

\section{Study design and medications (Fig. 1)}

After screening, all patients underwent a 2-week wash-out period in which no therapy was administered. After this period, patients were randomly assigned in a $1: 1$ ratio using computer randomization (StatsDirect Ltd., Cheshire, UK) to receive $2 \mathrm{ml}$ of tacrolimus $0.1 \%$ ointment (equivalent to $0.2 \mathrm{mg}$ of tacrolimus) or $2 \mathrm{ml}$ of clobetasol propionate $0.05 \%$ ointment (equivalent to $1 \mathrm{mg}$ of clobetasol) four times daily for 4 weeks. All therapy was discontinued after 4 weeks. Tacrolimus was prepared by a pharmacist (S. G.) at the Servizio Farmacia of the Policlinico "G.B. Rossi" of Verona, Italy. Clobetasol propionate $0.05 \%$ ointment was purchased from GlaxoSmithKline, Verona, Italy. Medications were identically packaged to maintain examiner and subject blinding.

Patients were instructed to apply the medications using a latex glove and to refrain from eating and drinking for at least $30 \mathrm{~min}$. after application. All patients maintained a diary throughout the study to assess compliance with therapy. No rescue medications were allowed during the study. One dental surgeon (G. C.) was responsible for ensuring randomization, blinding, and dispensing medications.

\section{Assessment of efficacy of the treatment}

Evaluations were performed at three time points: (1) before treatment (baseline), (2) 4 weeks after beginning treatment, and (3) 6 weeks after beginning treatment ( 2 weeks after discontinuing treatment). All clinical evaluations were performed by one member of the study team (M. L. P.) who was blinded to treatment assignment. The signs of OLP (i.e., mucosal extension) were quantified by this investigator using a calculated score based on the surface extension of mucosal lesions. This score was determined using a drawing in which the areas of various zones of affected mucosa were marked and expressed as a percentage of the total oral mucosa as previously described by Corrocher et al. (2006). Mucosal lesion extension was

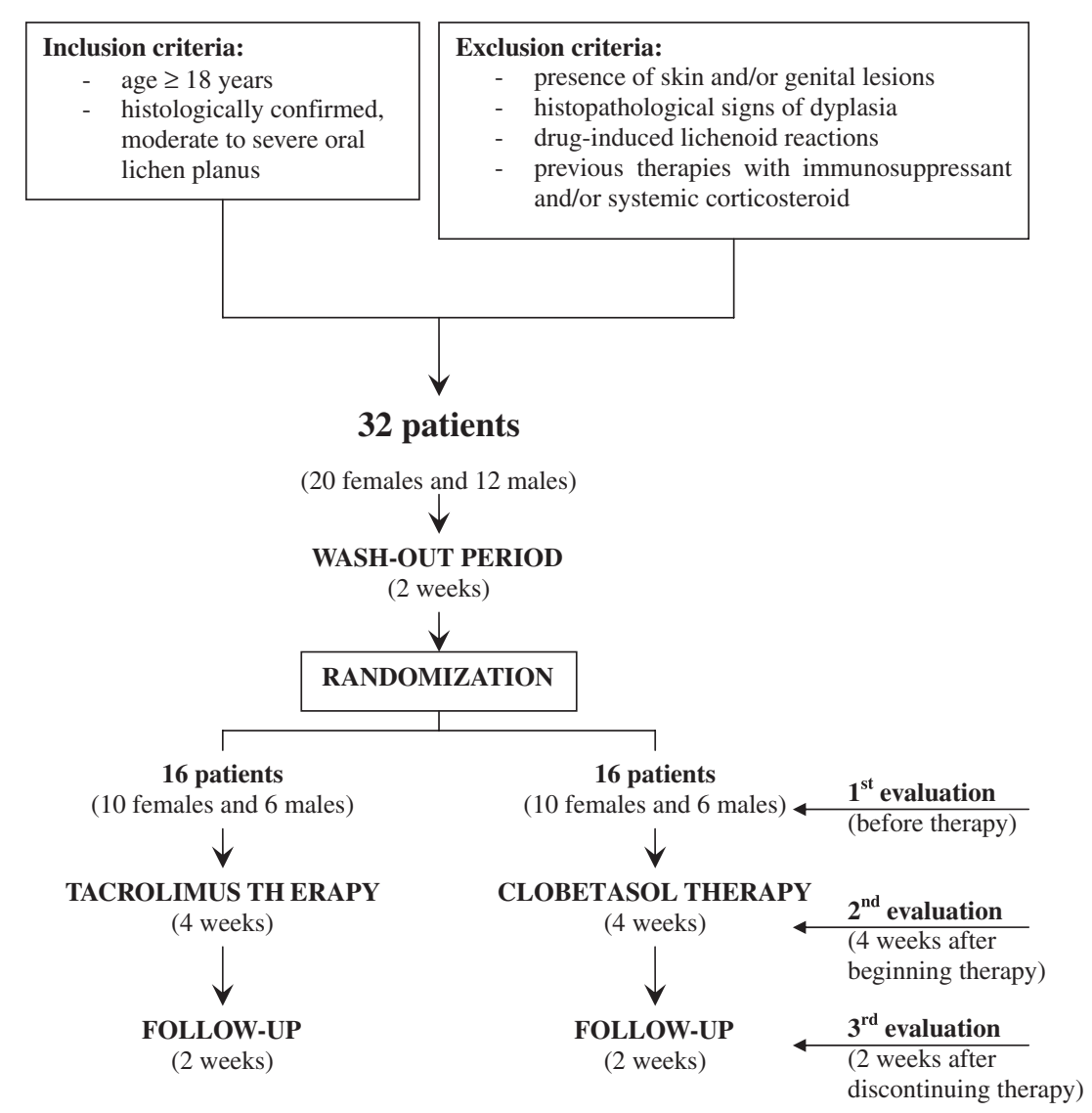

Fig. 1. Trial diagram. 
assessed using a four-point scale [based on a percentage estimate of the affected mucosa: $0=$ absent; $1=$ involvement $<5 \%$ of total mucosal surface area (mild); $2=5-15 \%$ of total mucosal surface area (moderate); and $3=>15 \%$ of total mucosal surface area (severe)]. Pain and burning sensation were also assessed using a four-point scale $(0=$ absent,$\quad 1=$ mild, $\quad 2=$ moderate , and $3=$ severe).

\section{Tolerability and safety assessment}

The tolerability of treatments was assessed using haematological and biochemical testing. At baseline, at the conclusion of treatment (4 weeks), and at the follow-up visit (6 weeks), patients underwent haematological testing (measurement of haemoglobin concentration and counts of red blood cells, platelets, and white blood cells, performed with a Technicon-H1 blood cell counter, Bayer, Leverkusen, Germany), electrolyte testing (measurement of serum sodium and potassium), kidney function testing (serum creatinine, serum urea, and urinary analysis), liver function testing (serum aspartate aminotransferase, serum alanine aminotransferase, serum alkaline phosphatase, and serum $\gamma$-glutamyl transpeptidase), and fasting plasma glucose testing (performed with an autoanalyser Modular AR, ISE 900 P800, Roche Diagnostics Systems, Basel, Switzerland). Blood concentrations of tacrolimus and its metabolites were measured using a commercial kit (Dade Behring Syva Enzyme Multiplied Immunoassay Technique, Liederbach, Germany) and a Cobas Integra 400 analyser (Roche Diagnostics Systems). The lower limit of detection in the serum was $1.5 \mathrm{ng} / \mathrm{l}$.

Adverse events were also recorded at each visit based on spontaneous reports by patients, patient interview, and/or direct observation by the investigator (M. L. P.). The severity of adverse events was assessed using a three-point scale (mild, moderate, or severe) and the relationship of each adverse event to treatment was assessed as unrelated, unlikely to be related, possibly related, probably related, or almost certainly related to study treatment.

\section{Microbiological procedures for Candida detection}

Secondary infection with Candida species that can exacerbate OLP symptoms is very common in OLP patients, especially those treated with topical corticosteroids. Therefore, unstimulated whole saliva from all patients was cultured for Candida using Sabouraud Dextrose Agar (Difco Laboratories, Detroit, MI, USA) at baseline, at the conclusion of treatment (4 weeks) and at the follow-up visit (6 weeks).

\section{Compliance}

Compliance was assessed by evaluating the unused drug at the end of the treatment period and by checking the daily diary of each patient about use of the assigned medication.

\section{Statistical analysis}

Statistical analysis was performed with the SPSS 13.0 software package (SPSS Inc., Chicago, IL, USA). Severity scores for pain, burning sensation, and mucosal extension were considered to be ordinal and were analysed using nonparametric tests. The proportions of symptom scores for each treatment group were analysed at each time interval (baseline, 4 weeks, 6 weeks) using the Wilcoxon signed-rank test. The Mann-Whitney $U$-test was used to statistically compare symptom scores among treatment groups at each time interval. The primary end point was the number of patients who achieved complete remission (severity score of 0 ) of symptoms/ signs. A difference of $50 \%$ in prevalence between the two treatment groups of this outcome was considered to be clinically meaningful. A sample size of 32 patients was calculated a priori for a power of $90 \%$ to detect this difference as determined by the Altman nomogram (Altman 1980).

\section{Results}

A total of 32 patients (20 females and 12 males; all white, Italian origin, with a mean age of $43.6 \pm 18.4$ years; 16 patients per treatment group) were enrolled in this study. The two treatment groups were not statistically different for gender distribution (tacrolimus and clobetasol group 6/16 males) and age (tacrolimus group mean age $=43.6 \pm$ 19.3 years versus clobetasol group mean age $=43.7 \pm 18.2$ years). All enrolled patients completed all phases of the trial, including the 2 -week followup visit (100\% compliance).
Table 1 gives the number and percentages of patients with various severity scores for pain, burning sensation, and mucosal extension at baseline, at the end of the 4-week treatment period, and at the 2-week follow-up visit (week 6) in each treatment group. At baseline, all patients in both treatment groups had pain, burning sensation, and mucosal extension with severity scores ranging from 2 to 3 . There were no statistically significant differences in the median symptom scores between the tacrolimus and clobetasol groups at baseline.

\section{Efficacy}

At the end of treatment, all patients in the tacrolimus group had decreased pain, burning sensation, and mucosal extension, with a substantial proportion of subjects achieving complete remission of symptoms/signs $(68.8 \%, 93.7 \%$, and $56.3 \%$, respectively); median 4 week scores were significantly less than median baseline scores for these parameters $(p<0.001)$. The improvement was maintained during follow-up ( $p<0.001$ versus baseline) for median burning sensation and mucosal extension scores, but we found that the median pain severity score was statistically significantly greater $(p<0.05)$ at the 6-week visit compared with the 4-week visit. Nonetheless, $43.7 \%$ of patients maintained complete pain remission in the tacrolimus group. In the clobetasol group, the proportion of patients with mild symptoms/signs for all parameters appeared to be greater at the end of treatment (week 4) and at the follow-up visit (week 6) than at baseline. The 4- and 6-week median pain and mucosal lesion extension scores were significantly lower than baseline median scores $(p<0.001)$. Only one patient $(6.3 \%)$ achieved complete remission of pain at 4 weeks and no patient achieved complete remission of burning sensation and mucosal extension. Furthermore, at the follow-up visit (week 6) in the clobetasol group, there was a slight, but statistically significant, increase of the median severity scores for all the three measured symptoms/ signs $(p<0.05)$ versus end of treatment (week 4). Importantly, at the end of treatment and at the follow-up evaluation, median pain, burning sensation, and mucosal extension symptom scores were significantly lower in the tacrolimus group compared with the clobetasol group $(p<0.001)$. 
Table 1. Number and percentage of patients with various severity scores for pain, burning sensation, and mucosal extension ${ }^{\dagger}$ among patients receiving tacrolimus $0.1 \%(0.2 \mathrm{mg})$ or clobetasol $0.05 \%(1.0 \mathrm{mg}) \mathrm{QD}$, for 4 weeks, for the treatment of oral lichen planus

\begin{tabular}{|c|c|c|c|c|c|c|}
\hline \multirow{2}{*}{$\begin{array}{l}\text { Symptoms/signs } \\
\text { and severity score }\end{array}$} & \multicolumn{3}{|c|}{ Tacrolimus $(n=16)$} & \multicolumn{3}{|c|}{ Clobetasol $(n=16)$} \\
\hline & $\begin{array}{l}\text { baseline } \\
\text { (week 0) }\end{array}$ & $\begin{array}{l}\text { end of } \\
\text { treatment } \\
\text { (week 4) }\end{array}$ & $\begin{array}{l}\text { follow-up } \\
\text { (week 6) }\end{array}$ & $\begin{array}{l}\text { baseline } \\
\text { (week 0) }\end{array}$ & $\begin{array}{l}\text { end of } \\
\text { treatment } \\
\text { (week 4) }\end{array}$ & $\begin{array}{l}\text { follow-up } \\
\text { (week 6) }\end{array}$ \\
\hline \multicolumn{7}{|l|}{ Pain } \\
\hline 3 (severe) & $12(75.0 \%)$ & 0 & 0 & $12(75.0 \%)$ & 0 & 0 \\
\hline 2 (moderate) & $4(25.0 \%)$ & 0 & 0 & $4(25.0 \%)$ & $5(31.2 \%)$ & $9(56.3 \%)$ \\
\hline 1 (mild) & 0 & $5(31.2 \%)$ & $9(56.3 \%)$ & 0 & $10(62.5 \%)$ & $7(43.7 \%)$ \\
\hline 0 (absent) & 0 & $11(68.8 \%)$ & $7(43.7 \%)$ & 0 & $1(6.3 \%)$ & 0 \\
\hline Median score (range) & $3(2-3)$ & $0(0-1)^{*}$ & $1(0-1)^{*, \#}$ & $3(2-3)$ & $1(0-2)^{*, \S}$ & $2(1-2)^{*, \#, \S}$ \\
\hline \multicolumn{7}{|c|}{ Burning sensation } \\
\hline 3 (severe) & $11(68.8 \%)$ & 0 & 0 & $11(68.8 \%)$ & 0 & 0 \\
\hline 2 (moderate) & $5(31.2 \%)$ & 0 & 0 & $5(31.2 \%)$ & $1(6.3 \%)$ & $7(43.7 \%)$ \\
\hline 1 (mild) & 0 & $1(6.3 \%)$ & $1(6.3 \%)$ & 0 & $15(93.7 \%)$ & $9(56.3 \%)$ \\
\hline 0 (absent) & 0 & $15(93.7 \%)$ & $15(93.7 \%)$ & 0 & 0 & 0 \\
\hline Median score (range) & $3(2-3)$ & $0(0-1)^{*}$ & $0(0-1)^{*}$ & $3(2-3)$ & $1(1-2)^{*, \S}$ & $1(1-2)^{*, \#, \S}$ \\
\hline \multicolumn{7}{|l|}{ Mucosal extension } \\
\hline 3 (severe) & $13(81.3 \%)$ & 0 & 0 & $13(81.3 \%)$ & 0 & 0 \\
\hline 2 (moderate) & $3(18.7 \%)$ & 0 & 0 & $3(18.7 \%)$ & $2(12.5 \%)$ & $9(56.3 \%)$ \\
\hline 1 (mild) & 0 & $7(43.7 \%)$ & $7(43.7 \%)$ & 0 & $14(87.5 \%)$ & $7(43.7 \%)$ \\
\hline 0 (absent) & 0 & $9(56.3 \%)$ & $9(56.3 \%)$ & 0 & 0 & 0 \\
\hline Median score (range) & $3(2-3)$ & $0(0-1)^{*}$ & $0(0-1)^{*}$ & $3(2-3)$ & $1(1-2)^{*, \wedge}$ & $2(1-2)^{*, \#, \S}$ \\
\hline Adverse events & 0 & $9(56.3 \%)$ & 0 & 0 & 0 & 0 \\
\hline
\end{tabular}

* $p<0.001$ versus baseline by Wilcoxon's signed-rank test.

$\# p<0.05$ versus end of treatment by Wilcoxon's signed-rank test.

${ }^{\S} p<0.001$ versus tacrolimus by Mann-Whitney test.

$\hat{p}<0.01$ versus tacrolimus by Mann-Whitney test.

'Severity score indicates the affected percentage of the whole surface of the gingival mucosa, ranging from 0 to $3: 0=$ absent; $1=$ involvement of $<5 \%$ of surface (mild); $2=5-15 \%$ (moderate); and $3=>15 \%$ (severe).

\section{Tolerability}

No significant changes in haematological and biochemical measures after 4 weeks of treatment were observed in either group and no severe adverse events were reported in either treatment group. In the group treated with tacrolimus, nine of 16 patients $(56.3 \%)$ reported an initial worsening of burning sensation during the first 2 days of treatment. However, it resolved rapidly within 4-5 days as OLP improved. No oral candidiasis was clinically or microbiologically observed in either group. After 4 weeks of treatment, all patients treated with tacrolimus had undetectable $(<1.5 \mathrm{pg} / \mathrm{l})$ serum concentrations of tacrolimus and its metabolites.

\section{Discussion}

In this study, topical tacrolimus $0.1 \%$ ointment was significantly more effective than topical clobetasol propionate $0.05 \%$ ointment for treatment of OLP. All the patients treated with tacrolimus $0.1 \%$ or clobetasol propionate $0.05 \%$ ointment had an improvement in symp- toms. Moreover, a substantial proportion of subjects treated with topical tacrolimus $0.1 \%$ ointment achieved complete remission of symptoms $(68.8 \%$, $93.7 \%$, and $56.3 \%$ for pain, burning sensation, and mucosal extension, respectively), while only one patient $(6.3 \%)$ treated with clobetasol achieved complete remission of pain and no patients treated with clobetasol had complete remission of burning sensation or mucosal lesion extension.

OLP is a chronic inflammatory mucocutaneous disease. The involvement of mucous membranes is frequent, but usually asymptomatic. However, mucosal involvement is occasionally complicated by extensive, painful erosions, causing a considerable decrease in quality of life. The oral mucosa, as well as the genital mucosa, conjunctiva, oesophagus, larynx, and anus, may be affected. In contrast to cutaneous lesions, erosive mucosal lichen planus is highly resistant to topical treatment and tends to have a chronic course with little tendency for spontaneous resolution. Erosive mucosal lichen planus is reported to have a slightly increased risk of malignant transformation (Setterfield et al. 2000, Edwards \& Kelsch 2002, Eisen 2002, de Moura Castro Jacques et al. 2003, Eisen 2003, Dissemond 2004, DeRossi \& Ciarrocca 2005, Lodi et al. 2005). Clobetasol propionate has been reported to be effective in some open trials in the treatment of OLP (Lozada-Nur et al. 1991, Campisi et al. 2004). More recently, a placebocontrolled trial compared clobetasol $0.05 \%$ ointment with fluocinonide $0.05 \%$ ointment in combination with antimycotic treatment, consisting of miconazole gel and $0.12 \%$ chlorhexidine mouthwashes. The results suggested that clobetasol was superior to fluocinonide in controlling OLP in most cases, without significant adrenal suppression or other adverse effects. Moreover, a concomitant antimycotic treatment with miconazole gel and chlorhexidine mouthwashes is a useful and safe prophylaxis against oropharyngeal candidiasis (Carbone et al. 1999). The usual treatment period with clobetasol is 2 weeks. However, longer treatment periods have been proposed for refractory disease (Edwards \& Kelsch 2002). Therefore, in order to attempt complete remission of symptoms, we choose a longer treatment period of 4 weeks. Some studies reported that topical tacrolimus is effective for the treatment of OLP (Olivier et al. 2002, Byrd et al. 2004, Laeijendecker et al. 2006). Laeijendecker et al. (2006) treated patients affected by OLP with tacrolimus $0.1 \%$ ointment, and found a relapse of symptoms in 13 of 18 patients within 3-9 weeks of treatment cessation.

Our trial further supports these observations and suggests that topical tacrolimus treatment may be more efficacious than high-potency topical corticosteroids (Kaliakatsou et al. 2002, Corrocher et al. 2006, Shichinohe et al. 2006). However, an abrupt discontinuation of topical corticosteroids may have resulted in some rebound effect in the clobetasol group during the follow-up period. On the other hand, the therapy with tacrolimus was clearly more efficacious than clobetasol during the treatment period.

The principal limitation of this study is the short duration of the treatment period, which was too brief to identify possible adverse events due to long-term use of the tacrolimus. It should be emphasized that a substantial proportion of patients in the tacrolimus group (9/16) had a diffuse sensation of oral 
mucosal burning at the beginning of treatment. However, this sensation was transient and well tolerated. Indeed, it was reported only during the first 2 days of treatment and was resolved rapidly within 4-5 days as OLP improved.

Another limitation of our study is the lack of a placebo control group. In this regard, the Human Subjects Committee of the Institutional Review Board of the Dipartimento di Medicina Clinica e Sperimentale of University of Verona would not approve the use of a placebo in these patients because the study had no provision for rescue medication. Moreover, a previous study (Carbone et al. 1999) demonstrated that topical steroids were beneficial in treating OLP and withholding effective therapy would not be ethical.

In conclusion, the results of this clinical trial suggest that topical tacrolimus $0.1 \%$ ointment is more effective than clobetasol propionate $0.05 \%$ ointment in the treatment of OLP. However, additional larger studies, with a longer treatment period and follow-up, are needed to confirm these findings before the use of tacrolimus ointment can be recommended in daily clinical practice.

\section{References}

Altman, D. G. (1980) Statistics and ethics in medical research III. How large a sample? BMJ 281, 1336-1338.

Byrd, J. A., Davis, M. D., Bruce, A. J., Drage, L. A. \& Rogers, R. S. III. (2004) Response of oral lichen planus to topical tacrolimus in 37 patients. Archives of Dermatology 140, 1508-1512.

Campisi, G., Giandalia, G., De Caro, V., Di Liberto, C., Arico, P. \& Giannola, L. I. (2004) A new delivery system of clobetasol-17propionate (lipid-loaded microspheres $0.025 \%$ ) compared with a conventional formulation (lipophilic ointment in a hydrophilic phase $0.025 \%$ ) in topical treatment of atrophic/ erosive oral lichen planus. A Phase IV, randomized, observer-blinded, parallel group clinical trial. British Journal of Dermatology 150, 984-990.

Carbone, M., Conrotto, D., Carrozzo, M., Broccoletti, R., Gandolfo, S. \& Scully, C. (1999) Topical corticosteroids in association with miconazole and chlorhexidine in the long-term management of atrophicerosive oral lichen planus: a placebocontrolled and comparative study between clobetasol and fluocinonide. Oral Diseases 5, 44-49.
Carbone, M., Goss, E., Carrozzo, M., Castellano, S., Conrotto, D., Broccoletti, R. \& Gandolfo, S. (2003) Systemic and topical corticosteroid treatment of oral lichen planus: a comparative study with long term follow-up. Journal of Oral Pathology and Medicine 32, 323-329.

Chan, E. S., Thornhill, M. \& Zakrzewska, J. (2000) Interventions for treating oral lichen planus. Cochrane Database of Systematic Reviews 2, CD001168.

Conrotto, D., Carbone, M., Carrozzo, M., Arduino, P., Broccoletti, R., Pentenero, M. \& Gandolfo, S. (2006) Ciclosporin vs. clobetasol in the topical management of atrophic and erosive oral lichen planus: a double-blind, randomized controlled trial. British Journal of Dermatology 154, 139-145.

Corrocher, G., Di Lorenzo, G., Mansueto, P., Martinelli, N., Esposito-Pellitteri, M., Gelio, S., Lombardo, G. \& Pacor, M. L. (2006) Comparison of topical tacrolimus $0.1 \%$ in pectin ointment with clobetasol $0.5 \%$ ointment in adults with moderate to severe desquamative gingivitis: a 4-week, randomized, double-blind clinical trial. Clinical Therapeutics 28, 1296-1302.

de Moura Castro Jacques, C., Cardozo Pereira, A. L., Cabral, M. G., Cardoso, A. S. \& Ramos-e-Silva, M. (2003) Oral lichen planus part I: epidemiology, clinics, etiology, immunopathogeny, and diagnosis. Skinmed 2, 342-347.

DeRossi, S. S. \& Ciarrocca, K. N. (2005) Lichen planus, lichenoid drug reactions, and lichenoid mucositis. Dental Clinics of North America 49, 77-89.

Dissemond, J. (2004) Oral lichen planus: an overview. Journal of Dermatologic Treatment 15, 136-140.

Edwards, P. C. \& Kelsch, R. (2002) Oral lichen planus: clinical presentation and management. Journal of the Canadian Dental Association 68, 494-499.

Eisen, D. (2002) The clinical features, malignant potential, and systemic associations of oral lichen planus: a study of 723 patients. Journal of the American Academy of Dermatology 46, 207-214.

Eisen, D. (2003) The clinical manifestations and treatment of oral lichen planus. Dermatology Clinic 21, 79-89.

European Agency for the Evaluation of Medicinal Products (2002) International Conference on Harmonisation-World Health Organization. Guideline for Good Clinical Practice [EMEA Web site]. ICH Topic E6. Geneva, Switzerland: WHO. Available at: http://www.emea.eu.int (accessed July 1996).

Kaliakatsou, F., Hodgson, T. A., Lewsey, J. D., Hegarty, A. M., Murphy, A. G. \& Porter, S. R. (2002) Management of recalcitrant ulcerative oral lichen planus with topical tacrolimus. Journal of the American Academy of Dermatology 46, 35-41.
Laeijendecker, R., Tank, B., Dekker, S. K. \& Neumann, H. A. (2006) A comparison of treatment of oral lichen planus with topical tacrolimus and triamcinolone acetonide ointment. Acta Dermato-Venereologica 86, 227-229.

Lodi, G., Scully, C., Carrozzo, M., Griffiths, M., Sugerman, P. B. \& Thongprasom, K. (2005) Current controversies in oral lichen planus: report of an international consensus meeting. Part 2. Clinical management and malignant transformation. Oral Surgery, Oral Medicine, Oral Pathology, Oral Radiology, and Endodontology 100, 164-178.

Lozada-Nur, F., Huang, M. Z. \& Zhou, G. A. (1991) Open preliminary clinical trial of clobetasol propionate ointment in adhesive paste for treatment of chronic oral vesiculoerosive diseases. Oral Surgery, Oral Medicine, Oral Pathology, Oral Radiology 71, 283-287.

Olivier, V., Lacour, J. P., Mousnier, A., Garraffo, R., Monteil, R. A. \& Ortonne, J. P. (2002) Treatment of chronic erosive oral lichen planus with low concentrations of topical tacrolimus: an open prospective study. Archives of Dermatology 138, 1335-1338.

Rozycki, T. W., Rogers, R. S. III, Pittelkow, M. R., McEvoy, M. T., el-Azhary, R. A., Bruce, A. J., Fiore, J. P. \& Davis, M. D. (2002) Topical tacrolimus in the treatment of symptomatic oral lichen planus: a series of 13 patients. Journal of the American Academy of Dermatology 46, 27-34.

Setterfield, J. F., Black, M. M. \& Challacombe, S. J. (2000) The management of oral lichen planus. Clinical and Experimental Dermatology 25, 176-182.

Shichinohe, R., Shibaki, A., Nishie, W., Tateishi, Y. \& Shimizu, H. (2006) Successful treatment of severe recalcitrant erosive oral lichen planus with topical tacrolimus. Journal of European Academy of Dermatology and Venereology 20, 66-68.

Sloberg, K., Hersle, K., Mobacken, H. \& Thilander, H. (1983) Severe oral lichen planus: remission and maintenance with vitamin A analogues. Journal of Oral Pathology 12, 473-477.

World Medical Association Declaration of Helsinki: Recommendations Guiding Medical Doctors in Biomedical Research Involving Human Subjects [WMA Web site]. FerneyVoltaire, France: WMA; 1989. Available at: http://www.wma.net/e/policy/b3.htm.

Accessed October 2004.

\section{Address:}

Prof. Gabriele Di Lorenzo

Dipartimento di Medicina Clinica e delle

Patologie Emergenti

Università di Palermo

Via del Vespro

141 -90127 Palermo

Italy

E-mail: dilo601@unipa.it 


\section{Clinical Relevance}

Scientific rationale for the study: OLP is a chronic inflammatory disease, characterized by local pain and burning sensation. Therapeutic options include high-potency topical corticosteroids and the use of immunosuppressive drugs.

Principal findings: In this study, topical $0.1 \%$ tacrolimus ointment was significantly more effective than topical $0.05 \%$ clobetasol ointment in relieving patient symptoms. All patients treated with tacrolimus or clobetasol had an improvement in symptoms and a substantial proportion of subjects treated with tacrolimus achieved complete remission of symptoms.
Practical implications: Topical tacrolimus appeared to be more effective than topical clobetasol for the treatment of OLP, but larger studies with more subjects, a longer treatment period, and longer followup are needed before the use of tacrolimus ointment can be recommended in clinical practice. 